\title{
Oficinas de Robótica para alunos do Ensino Médio: introduzindo a computação para futuros ingressantes
}

\author{
Bianca Alessandra Visineski Alberton ${ }^{1}$, Marília Abrahão Amaral ${ }^{1,2}$ \\ ${ }^{1}$ Departamento Acadêmico de Informática - Universidade Tecnológica Federal do \\ Paraná (UTFPR) \\ ${ }^{2}$ Programa de Pós-graduação em Tecnologia e Sociedade (PPGTE) - Universidade \\ Tecnológica Federal do Paraná (UTFPR) \\ bi.alberton@gmail.com, mariliaadutfpr.edu.br
}

\begin{abstract}
This paper describes the development and implementation of a workshop on introduction to robotics designed for high school students. The initiative is an activity of teaching and extension of the group PET Computando Culturas em Equidade and it is intended to spread computing and robotics in high school as a way of attracting prospects students in computing-related courses.
\end{abstract}

Resumo. Este artigo descreve a elaboração e execução de um oficina de introdução à robótica destinada a alunos do ensino médio. Tal iniciativa trata-se de uma atividade de ensino e extensão do grupo PET Computando Culturas em Equidade e é destinada a difundir a informática e a robótica entre os alunos do ensino médio, como uma forma de atrair futuros ingressantes nos cursos relacionados à computação.

\section{Introdução}

Diversas pesquisas trabalham com iniciativas para novos métodos de ensino de programação (Gonçalves, 2011), (Priesnitz, 2012), (Cardoso, 2001). Os trabalhos de Albuquerque (2007), Barbosa (2009), Miranda (2007), Pio (2006), D’Abreu (2001), Vilhete (2002), Queiroz (1998), Miranda (2010) trazem a robótica como componente do ensino de programação retomam os aspectos construtivistas (Piaget, 1990), (Lévy, 1987), (Papert, 1985) destas práticas de ensino.

Este trabalho apresenta a experiência de um grupo PET (Programa de Educação Tutorial), estruturado em consonância às afirmativas de inclusão do edital PETConexões de Saberes. A proposta do grupo PET-CoCE (Computando Culturas em Equidade) é talhada para estreitar os laços entre a universidade e a sociedade, oportunizando atividades relacionadas a ensino, pesquisa e extensão para uma população tradicionalmente com acesso restrito a este tipo de formação, sobretudo qualificando o retorno destas formações a diferentes espaços e movimentos sociais. Nesta equipe participam estudantes dos seguintes cursos: Bacharelado em Sistemas de Informação, Bacharelado em Design, Engenharia da Computação, Engenharia Eletrônica e Engenharia Mecânica.

O grupo possui forte articulação com o Departamento Acadêmico de Informática, da Universidade Tecnológica Federal do Paraná (UTFPR), e os cursos de 
Bacharelado em Sistemas de Informação e Engenharia da Computação que são oferecidos por colegiado interdisciplinar e envolvem atividades afirmativas que englobam a construção de subjetividades pelo aprendizado participativo (educação participativa para a liberdade - ensino), a sistematização e o amadurecimento sóciotécnico, cultural e profissional (pesquisa) e o fomento a intervenções situadas de relevância social, correntes e futuras (extensão).

As atividades do PET-CoCE primam por incentivar a participação de minorias na computação. Estão sendo desenvolvidas oficinas para atender alunos e alunas do ensino médio, provenientes da rede pública de ensino, alunos e alunas participantes do projeto "Sala de Altas Habilidades e Superdotação" do Instituto de Educação do Paraná Professor Erasmo Pilotto (IEPPEP), servidores da Universidade Tecnológica Federal do Paraná (UTFPR) e alunas do IEPPEP em atividades específicas para inclusão de mulheres na computação.

Um dos vieses de atividades desenvolvidas pelo PET-CoCE, são as oficinas de ensino, que tratam de tópicos relacionados à computação. Uma delas é denominada "Oficina de Introdução à Robótica e Utilização de Sensores com Arduino".

O objetivo desta oficina é difundir a robótica no ensino médio, proporcionando aos alunos trabalhar com componentes básicos, tais como microcontroladores, motores, circuitos integrados e sensores, bem como desmistificar as atividades dos profissionais da área de computação. Busca-se ainda difundir uma robótica de baixo custo e com maior entendimento do funcionamento dos componentes utilizados, constituindo-se uma alternativa à utilização de kits educacionais comerciais, como o LEGO, amplamente difundidos nas instituições que trabalham com robótica educacional.

Nesse sentido, emprega-se nessa oficina o kit microcontrolado Arduino ${ }^{1}$, que possui uma interface amigável ao usuário e vem sendo utilizados por profissionais de diversas áreas do conhecimento, como designers, mecânicos, etc. Devido ao fato de a maioria dos alunos do ensino médio não conhecerem nenhuma linguagem de programação, o grupo PET-CoCE também oferece a oficina de "Fundamentos de Programação e Eletrônica Baseados na plataforma Arduino", na qual são abordados conceitos básicos de programação como utilização de variáveis, funções, estruturas condicionais (if-else e switch-case) e estruturas de repetição (for e while).

Este artigo tem como objetivo descrever o processo de condução das atividades e os resultados obtidos com a oficina proposta. Para tal o documento foi dividido em 5 seções. A primeira apresenta a introdução. A segunda seção aborda a motivação para o desenvolvimento da pesquisa. A terceira seção apresenta a metodologia utilizada para o desenvolvimento das oficinas. Já na seção de número quatro são apresentados detalhes sobre os projetos propostos. Na quinta seção são descritas as considerações finais. Por fim, estão os agradecimentos e as referências.

\section{Motivação}

A necessidade de engenheiros, no país, é extremamente elevada e altamente relevante e, agravando esta situação, os cursos de engenharia possuem um alto grau de

\footnotetext{
${ }^{1}$ Plataforma de prototipagem eletrônica de hardware livre. Disponível em: <http://arduino.cc/>.
} 
desistência. A engenharia brasileira pode não dar conta do volume de investimentos planejados para os próximos anos, já que, de acordo com a Federação Nacional dos Engenheiros (FNE), são formados apenas cerca de 20 mil engenheiros por ano (LIMA, 2010). Esta demanda não é suficiente para satisfazer ao mercado.

O diagnóstico da realidade nos 1.374 cursos no país mostra que a evasão nos cursos de engenharia é de $80 \%$; dos 150 mil que ingressam no primeiro ano, 30 mil se formam (BRITO, 2010). Desta forma, percebe-se que os cursos de engenharia, embora muito procurados, formam um contingente muito pequeno, proporcionalmente. Segundo Pereira et al. (2006), uma das hipóteses para esta desistência é falta de perspectiva de onde será usado todo o ferramental básico nas disciplinas posteriores.

Assim, a introdução de artefatos que demonstrem aplicação das ferramentas apresentadas inicialmente nos cursos de engenharia, é uma alternativa para evitar que alunos dos primeiros períodos destes cursos desistam por não terem suas expectativas atendidas nas disciplinas básicas.

Para complementar a hipótese apresentada anteriormente, para a desistência dos alunos dos primeiros semestres dos cursos de engenharia, pode-se citar o artigo Simas (2012), o qual aponta que muitos alunos desistem de seus cursos devido ao fato de só perceberem que fizeram a escolha do curso errado após iniciarem este, ou ainda, devido às disciplinas práticas estarem presentes entre a metade e o fim do curso, fazendo com que os alunos desanimem.

Entende-se por "escolha do curso errado" a falta de informação sobre o curso, instituição e até mesmo da profissão em que os alunos irão exercer após a formação. Os recém-ingressos são muitas vezes movidos por expectativas infundadas a respeito dos pontos citados anteriormente, levando-os à decepção sobre a escolha e à evasão (MARINS et al., 2010).

Em seu artigo, Oliver et al. (2010) afirmam que já foram lançadas iniciativas que aplicam a técnica de PBL (Project Based Learning ${ }^{2}$ ) aliada à robótica como modo de motivar os alunos dos cursos de Engenharia de Computação e Ciência da Computação. De acordo com eles, os robôs podem ser utilizados para o aprendizado tanto das disciplinas básicas, como programação, quanto das mais avançadas, tais como Inteligência Artificial e Engenharia de Software.

Desta forma, diversas disciplinas dos cursos de graduação em computação da UTFPR estão relacionadas com a robótica, mas encontram-se, em sua maioria, ao final do curso, tais como Sistemas Inteligentes 1 e 2 e Engenharia de Software, que estão presente tanto em Engenharia de Computação quanto em Bacharelado em Sistemas de Informação.

Além disso, em engenharia de Computação também há disciplinas de "Sistemas Microcontrolados" e "Sistemas Embarcados" (BASTOS et al., 2006), enquanto em Sistemas de Informação, há disciplinas relacionadas à administração e gestão, vertente que atua em desenvolvimento de projetos e de software, tais como Teoria Geral da Administração, Gerência de Projetos e Gestão da Informação e de Sistemas de Informação. Assim, a integração com estudantes de Engenharia de Computação mostra-

\footnotetext{
${ }^{2}$ Em português, aprendizado baseado em projetos.
} 
se de bastante valor, de forma que a organização e administração para gerenciamento dos projetos desenvolvidos seja otimizada, pois um dos objetivos do curso de Sistemas de Informação é formar estudantes com habilidades para desempenhar funções como a de gerente de tecnologia da informação e consultor/auditor na área de infraestrutura (GRAELM et al., 2008).

Desse modo, ao proporcionar aos alunos do ensino médio e a recém-ingressantes nos cursos de computação um contato com um projeto realizado pelos graduandos auxiliaria a diminuir a evasão destes cursos, pois estes esclareceriam a questão sobre as formas que o conhecimento teórico poderia ser aplicado ao mesmo tempo em que motivariam os alunos a permanecerem no curso até chegar às disciplinas práticas, que se encontram nos últimos períodos. Além disso, esta iniciativa demonstra algumas vertentes que o estudante pode seguir em sua carreira profissional.

\section{Metodologia}

A oficina começou a ser projetada considerando seu público-alvo (alunos do ensino médio, em particular os integrantes da Sala de Altas Habilidades e Superdotação). Estes alunos participam de um projeto do IEPPEP que oferece atividades, no contra turno, para estudantes com altas habilidades de acordo com o conceito de inteligências múltiplas (Gardner, 1989). O horário das aulas, linguagem do material e a postura dos instrutores devem ser compatíveis com estes alunos. A partir disto, foi desenvolvido um material didático, em língua portuguesa, que pretende ser instrutivo e compreensivo, no sentido de ser autossuficiente, sem necessitar de conhecimentos e/ou leituras anteriores. Tal material didático constitui-se em uma apostila que é entregue gratuitamente aos alunos e em slides que estão disponíveis na página do grupo PET-CoCE.

Devido à grande procura de cursos desse gênero pelos alunos do ensino médio com curso técnico integrado da própria UTFPR, foram abertas cinco vagas, das doze ofertadas, especificamente para este público. Apesar de possuírem características diferentes dos alunos da Sala de Altas Habilidades, optou-se por não modificar o material didático em função deles, já que o material atende às demandas deste público também.

De modo a dar um maior enfoque às aplicações na robótica, é exigido dos alunos um conhecimento básico prévio de programação, seja obtido em outras oficinas ofertadas pelo PET- CoCE, seja obtido em outros lugares. Entretanto, a primeira aula é dedicada ao nivelamento dos mesmos quanto à programação básica e à estrutura da linguagem Wiring, que é baseada em $\mathrm{C} / \mathrm{C}++$ e é a utilizada na plataforma Arduino.

Na elaboração da oficina foi realizada uma seleção dos enunciados de projetos (atividades práticas utilizando o dispositivo Arduino), de diferentes complexidades, nas quais se abordam diversos conceitos de programação, como variáveis, funções, estruturas condicionais e de repetição.

Como o público-alvo é formado majoritariamente por alunos do $1^{\circ}$ e $2^{\circ}$ anos do ensino médio, incluiu-se no cronograma uma aula introdutória a conceitos de eletricidade, necessários para a melhor compreensão dos componentes eletrônicos.

Para cada componente utilizado na oficina, há uma seção na apostila explicando o funcionamento geral do mesmo, bem como uma possível montagem de circuito e um 
código básico para fazê-lo funcionar com o Arduino. Como diagramas esquemáticos não são intuitivos o suficiente para pessoas que nunca os viram, optou-se por mostrar imagens de como o circuito deve ser montado com o software open-source Fritizing ${ }^{3}$. A Figura 1 mostra uma possível montagem de um circuito para controle de um servo motor.

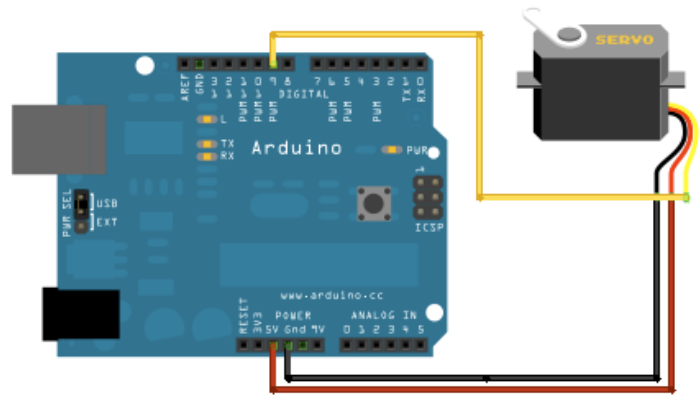

Figura 1 - Circuito de exemplo para funcionamento de um servo motor.

As aulas foram estruturadas de modo que houvesse primeiramente uma exposição do funcionamento básico de cada componente utilizado. Baseados no circuito e código fonte básico de utilização do componente, os alunos realizam testes para ver como os componentes funcionam. Após eles terem compreendido como eles funcionam, propõe-se um projeto comum a todos, onde cada equipe tem a oportunidade de praticar seus conhecimentos e trocar ideias com as outras equipes.

A oficina é introdutória à robótica, portanto não são abordados sensores de maior complexidade ou comunicação sem fio. Entretanto, a última aula da oficina é dedicada para apresentar aos alunos estes outros componentes, de modo a mostrar os diversos projetos que podem ser desenvolvidos dentro da robótica.

Ao final de cada aula, os alunos guardam o material utilizado, levando consigo apenas a apostila - os componentes eletrônicos permanecem sob os cuidados do grupo PET para uso em outros projetos e outras oficinas muitas vezes desenvolvidas em paralelo a essa.

Os kits utilizados são idênticos, sendo o kit básico de eletrônica composto por protoboard, resistores, diodos emissores de luz (LED), potenciômetros, buzzer, botões (ou chave tátil), resistores dependentes de luz (LDR), sensor de temperatura, alicates de corte e de bico, fios de cobre, além do Arduino e um cabo USB. Estes materiais são compartilhados com a oficina de "Fundamentos de Programação e Eletrônica Baseados na plataforma Arduino". Os demais componentes são fornecidos à medida que os alunos necessitarem para o desenvolvimento dos projetos e consistem em servo motores, sensores de distância por ultrassom, chassis com motores e caixas de redução, circuitos integrados L293NE (correspondente à estrutura denominada "ponte H"), diodos 1N4001, reguladores de tensão e uma bateria.

As aulas acontecem uma vez por semana, com duração de uma hora e meia, com um total de 12 encontros. Para receber o certificado de participação da oficina é exigida

${ }^{3}$ Disponível em: http://fritzing.org 
dos alunos uma frequência de presença igual ou superior a $75 \%$ das aulas dadas e nota maior ou igual à 7.0. A nota é composta por listas de exercícios e relatórios dos projetos desenvolvidos. Tal exigência foi criada tanto para testar se os alunos estão assimilando o conteúdo, quanto para proporcionar a eles um maior contato com o sistema acadêmico da engenharia.

\section{Projetos Propostos}

A oficina é organizada em dois grandes projetos: o robô seguidor do sol e o carrinho que desvia de objetos. O primeiro consiste na simulação de um dispositivo de captação de luz que segue o sol utilizando placas de papelão no lugar de células fotovoltaicas.

O segundo trata-se de um robô totalmente autônomo que se locomove por um ambiente sem colidir com os objetos à sua frente. Ele é desenvolvido em duas etapas: após aprender o funcionamento das baterias, reguladores de tensão, ponte $\mathrm{H}$ e motores DC, os alunos montam o circuito que lhes é dado e desenvolvem um código no qual o robô locomova-se para frente e para trás e seja capaz de fazer curvas para direita e para a esquerda. Na segunda etapa, os alunos aprendem o funcionamento do sensor de distância e o adicionam ao carrinho, complementando o código para que o robô não colida com os obstáculos.

\subsection{Projeto 1: Robô seguidor do sol}

De modo a estimular os alunos a desenvolverem projetos que resolvam problemas do mundo real, o enunciado proposto contextualiza a energia elétrica no Brasil e a necessidade de utilizar fontes de energia menos impactantes, como a geração de energia elétrica a partir da captação de luz solar com células fotovoltaicas. Uma solução para aumentar essa captação é fazer com que a placa esteja sempre voltada em direção ao sol.

Assim, por meio de sensores de luminosidade é possível saber em qual região a intensidade luminosa é maior e girar a placa para esta direção.

Utilizando placas de papelão no lugar de células fotovoltaicas, o robô é desenvolvido utilizando um servo motor, três sensores de luminosidade, resistores, cabos, uma protoboard e o Arduino. A disposição dos componentes fica por conta de cada equipe, bem como o código para desenvolvê-lo.

Como base deste projeto, os alunos recebem o circuito e código do funcionamento do servo motor que eles deverão utilizar e de um sensor operando sozinho. A Figura 2 mostra a montagem básica de um circuito utilizando o sensor de luminosidade (LDR). 


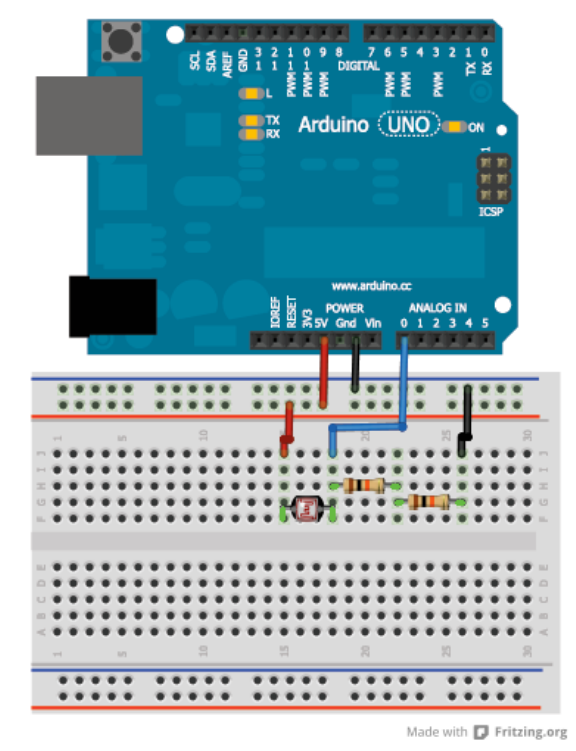

Figura 2 - Montagem básica de um circuito com LDR.

A resolução deste projeto é relativamente simples, bastando que sejam feitas comparações para verificar qual dos três sensores está captando mais luz e mover o servo motor naquela direção. Para isto utiliza-se a estrutura condicional if-else, funções da plataforma Arduino para leitura dos sensores e funções da biblioteca que modela os servo motores.

\section{Considerações Finais}

A oficina encontra-se hoje na sua segunda edição. Para ela foram realizadas modificações no material de modo que a linguagem estivesse mais clara. Verificou-se, por exemplo, que mostrar um código mais elaborado para os alunos os deixa confusos na hora de montar o projeto. Por exemplo, ao apresentar um código de utilização do LDR cuja função era classificar a leitura obtida em quatro níveis de luminosidade (escuro, breu, com intensidade média de luminosidade e claro) fez com que os alunos achassem que era necessário realizar esta operação para executar o projeto "Robô seguidor do Sol", enquanto bastava apenas ler o valor do sensor e comparar os valores obtidos para determinar qual sensor estava recebendo mais luz. Por isso procurou-se deixar os códigos base o mais simples possível.

Embora se considere que os alunos compreenderam o funcionamento básico dos componentes, verificou-se que certas abordagens utilizadas não são tão eficazes. De maneira geral, eles compreendem melhor o funcionamento dos componentes se a explicação for mais superficial e sem muitos detalhes. Este fato deve-se principalmente ao fato de muitos alunos não compreenderem muito os conceitos de física envolvidos, que vão desde a mecânica dos motores até a eletricidade aplicada na eletrônica.

$\mathrm{Na}$ atual edição da oficina observou-se que a procura pela oficina foi muito maior entre os alunos do ensino médio técnico da universidade do que entre os alunos da Sala de Altas Habilidades. Entretanto, observa-se que a oficina realmente interessa aos alunos, visto que o número de inscrições é muito superior ao número de vagas em ambas as edições da oficina. 
Na primeira edição foi constatada a evasão de $33 \%$ dos participantes. No início dessa última edição havia 12 alunos matriculados, o que caracteriza o preenchimento do total de vagas oferecidas. Um dos matriculados não compareceu em nenhuma aula e também não justificou o porquê de tal atitude. Os outros três desistentes encontraram problemas com o horário da oficina, que teve seu horário alterado uma vez ao longo do curso.

Dos concluintes da oficina, dois não receberam o certificado por não alcançarem a nota mínima. Entretanto, eles estão participando da atual edição da oficina, que está contando com 14 alunos.

Ao final da oficina foi enviado aos alunos um questionário online anônimo para registrar a opinião sobre a oficina. Participaram dele seis alunos, dos quais dois não concluíram a oficina por incompatibilidade de horários. Todos os alunos afirmaram que estavam satisfeitos com a oficina e $67 \%$ dos entrevistados a consideram de fácil entendimento. Além disso, $50 \%$ dos alunos alegaram não ter dedicado tempo extra classe para a oficina.

Quanto ao método de avaliação da oficina e o material didático utilizado, todos os alunos os avaliaram com uma nota de 4 a 5 , em que a nota máxima é 5 . Ao final do questionário, um dos alunos que concluiu a oficina e a considerou de fácil entendimento deixou o seguinte comentário: "O material didático é muito bom, mas os exercícios são simples e acabamos não exercitando tanto os conteúdos aprendidos”.

Considera-se que a oficina de robótica teve um grande impacto sobre os alunos, visto que alguns demonstraram grande interesse em participar de competições, como a Olímpiada Brasileira de Robótica ${ }^{4}$, enquanto outros buscaram desenvolver seus próprios projetos fora de sala de aula.

As oficinas ministradas pelo PET-CoCE mostram efeitos positivos advindos dos desdobramentos de uma interação de mão dupla com a sociedade, que conduz a um impacto nas formações, tanto de ensino médio como superior, e no exercício profissional.

Esta iniciativa se preocupa em pavimentar e tratar o processo de desmistificação da computação não somente com a formação de usuários e usuárias das tecnologias de informação e comunicação, mas sim com as condições para que a comunidade atendida seja desenvolvedora de artefatos computacionais e que desta forma possam exercer a prática de saberes e fazeres por meio das oficinas.

Espera-se que as atividades desenvolvidas nestas oficinas funcionem como interfaces entre a educação tradicionalmente oferecida em disciplinas com questões ligadas a dimensões sociais, culturais, históricas ou mesmo pessoais, aproximando as formações na área de computação e o exercício profissional das demandas e lacunas sociais tão merecedoras de atenção em nossa sociedade.

\footnotetext{
${ }^{4}$ Para mais informações acessar http://www.obr.org.br/.
} 


\section{Agradecimentos}

Este trabalho foi financiado pelo Programa de Educação Tutorial Conexões de Saberes, do Ministério da Educação (MEC), das Secretaria de Educação Superior (SESu) e Secretaria de Educação Continuada, Alfabetização e Diversidade (SECAD), aprovado em 2010.

\section{Referências}

Albuquerque A.P., Melo C.M., César D.R., Mill. D. (2007). Robótica Pedagógica Livre: Instrumento de Criação, Reflexão e Inclusão Sócio-Digital. Anais do Simpósio Brasileiro de Informática na Educação. Disponível em: <http://www.brie.org/pub/index.php/sbie/article/view/682>.

Arduino. (2013). Disponível em: <http://arduino.cc/>. Acesso em: 24 abr. 2013.

Barbosa M.R.G., Silva F.A., Oliveira V.M. de A., Feltrim V.D., Mirisola L.G.B., Gonçalves P.C., et al. (2009). Implementação de Compilador e Ambiente de Programação Icônica para a Linguagem Logo em um Ambiente de Robótica Pedagógica de Baixo Custo. Anais do Simpósio Brasileiro de Informática na Educação Disponível em: <http://www.brie.org/pub/index.php/sbie/article/view/1186>.

Bastos, L. C.; Merkle, L. E.; et al. (2006) Projeto Pedagógico do Curso de Engenharia de Computação. Disponível em: <http://www2.dainf.ct.utfpr.edu.br/ec/o-curso/ProjetoPedagogico.pdf>. Acesso em: 13 mai. 2013.

Brito, A. (2010). País perde US\$ 15 bi com má formação de engenheiro. Disponível em: <http://wwwl folha.uol.com.br/fsp/mercado/me2106201001.htm>. Acesso em: 25 abr. 2013.

D' Abreu, J.V.V, Chella, M.T. Ambiente Colaborativo de Aprendizagem a Distância Baseado no Controle de Dispositivos Robóticos. Anais do Simpósio Brasileiro de Informática na Educação. Disponível em: <http://www.br-ie.org/pub/index.php/sbie /article/view/145>.

Gardner, H., Hatcb, T. (1989). Multiple intelligences go to school: educational implications of the theory of Multiple Intelligences. Educational Researcher, v.18, n.8. p.4-10, 1989.

Gonçalves, R. E. F. (2011). Jogo digital para o ensino dos fundamentos da programação, Tese de mestrado integrado. Engenharia Informática e Computação. Universidade do Porto. Faculdade de Engenharia. 2011.

Graelm, A. R. et al. (2008). Projeto de Abertura do Curso Bacharelado em Sistemas de Informação. Disponível em: <http://www.dainf.ct.utfpr.edu.br/adolfo/ WikiDainf/ProjetoBSI- Final 02092008 V3.pdf>. Acesso em: 13 mai. 2013.

Lévy, Pierre. (1987). A máquina universo: Criação, cognição e cultura informática. Lisboa: Instituto Piaget, 1987.

Lima, F. (2010). Efeito cascata no ensino da engenharia. 2010. Disponível em: $<$ http://www.osetoreletrico.com.br/web/colunistas/carla-estremes-ricci/497-efeitocascata-no-ensino-da-engenharia.html>. Acesso em: 25 abr. 2013. 
Marins, C. N. M.; Côrrea, E. M.; Santana, R. G. (2010). Iniciação à Engenharia - Um programa para a diminuição da evasão de alunos. Disponível em: $<$ http://www.abenge.or.br/Cobenge Anteriores/2010/artigos/407.pdf>. Acesso em: 26 abr. 2013.

Miranda, L.C. de, Sampaio, F.F., Borges, J.A. dos S. (2007). ProgrameFácil: Ambiente de Programação Visual para o Kit de Robótica Educacional RoboFácil. Anais do Simpósio Brasileiro de Informática na Educação. Disponível em: <http://www.brie.org/pub/index.php/sbie/article/view/575>.

Miranda, L. C. de, Sampaio FF, Borges JA dos S. (2010). RoboFácil: Especificação e Implementação de um Kit de Robótica para a Realidade Educacional Brasileira. Revista Brasileira de Informática na Educação. Disponível em: <http://www.brie.org/pub/index.php/rbie/article/view/1275>.

Oliver, J.; Toledo, R.; Valderrama, E. (2010). A Learning Approach Based on Robotics in Computer Science and Computer Engineering. IEEE EDUCON Education Engineering 2010 - The Future of Global Learning Engineering Education.

Papert, Seymour. (1985). LOGO: Computadores e Educação. São Paulo: Editora Brasiliense, 1985.

Pereira, M. C. et al. (2006). Evitando evasão em cursos de engenharia: ume estudo de caso. Disponível em: <http://www.abenge.org.br/CobengeAnteriores/2006/artigos /1253802.pdf>. Acesso em: 25 abr. 2013.

Piaget, Jean. (1990). A formação do símbolo na criança: imitação, jogo e sonho, imitação e representação. Rio de Janeiro: LTC, 1990.

Pio, J.L. de S, Castro, T.H.C. de, Júnior, A.N. de C. (2006). A Robótica Móvel como Instrumento de Apoio à Aprendizagem de Computação. Anais do Simpósio Brasileiro de Informática na Educação. Disponível em: Available from: $<h t t p: / / w w w . b r$-ie.org/pub/index.php/sbie/article/view/510>.

Priesnitz Filho W, Abegg I, Simonetto E de O. (2012), Uma Abordagem Diferenciada no Ensino de Algoritmos Através da Utilização de uma Lousa Digital, GEINTEC Gestão, Inovação e Tecnologias, vol. 2, no. 2, pp. 129-137, 2012.

Queiroz, L.R. de, Bergerman, M., Machado, R.C., Bueno, S.S., Elfes, A. (1998). Educação a Distância em Robótica e Visão Computacional. Revista Brasileira de Informática na Educação. Disponível em: <http://www.br-ie.org/pub/index. php/rbie/article/view/2299>.

Simas, A. (2012). As graduações campeãs de desistência. Gazeta do Povo. Disponível em: <http://www.gazetadopovo.com.br/vidauniversidade/nocampus/conteudo.phtml? $i d=1248860 \&$ tit $=$ As-graduacoes-campeas-de-desistencia $>$. Acesso em: 23 abr. 2013.

Vilhete, J, Gonçalves, L.M.G, Garcia, M.F., Garcia, L.T.S. (2002) Uma Abordagem Prático-Pedagógica para o Ensino de Robótica em Ciência e Engenharia de Computação. Anais do Simpósio Brasileiro de Informática na Educação. Disponível em: <http://www.br-ie.org/pub/index.php/sbie/article/view/205>. 